Aki Asanuma • Toshihiro Ohura • Eishin Ogawa

Sachiko Sato • Yutaka Igarashi • Yoichi Matsubara

Kazuie Iinuma

\title{
Molecular analysis of Japanese patients with steroid 21-hydroxylase deficiency
}

\begin{abstract}
We have designed a rapid and convenient strategy to determine nine of the most common mutations in the 21-hydroxylase gene (CYP21). The frequency of the mutations was investigated in 34 Japanese patients affected with congenital adrenal hyperplasia (CAH) caused by 21hydroxylase deficiency. We characterized $82 \%$ of the $\mathrm{CAH}$ chromosomes. The most frequent mutations were a C/A to $\mathrm{G}$ substitution in intron 2 in the salt-wasting form of the disease and an $I 172 N$ in the simple virilizing form. Three de novo mutations were found. Two homozygous mutations (S268T and N493S) were detected by direct sequencing of all exons of $C Y P 21$ in two siblings, who had a normal genotype at all positions screened. We successfully applied these methods for prenatal diagnosis in one family. These procedures proved to be sensitive and rapid for the detection of the most common known mutations in the CYP21 gene and may be useful for genetic screening.
\end{abstract}

Key words Genetic screening · Genetic disease · 21Hydroxylase deficiency $\cdot$ PCR

\section{Introduction}

Congenital adrenal hyperplasia (CAH; MIM *201910) is one of the most common forms of inborn errors of metabolism. Steroid 21-hydroxylase (21-OH) deficiency is a cortisol biosynthesis autosomal recessive disorder that accounts for $90 \%-95 \%$ of CAH cases. A wide spectrum of clinical variants exists, from severe or classical forms, which are evident

A. Asanuma $\cdot$ T. Ohura $(\triangle) \cdot$ E. Ogawa $\cdot$ S. Sato $\cdot$ K. Iinuma Department of Pediatrics, Tohoku University School of Medicine, 1-1 Seiryo-machi, Aoba-ku, Sendai 980-8574, Japan

Tel. +81-22-717-7286; Fax +81-22-717-7290

e-mail: tohura@ped.med.tohoku.ac.jp

Y. Igarashi

Igarashi Childrn's Clinic, Sendai, Japan

Y. Matsubara

Department of Medical Genetics Tohoku University School of

Medicine, Sendai, Japan at birth, to mild, late-onset nonclassical forms. Severe forms include two groups of patients: those with a complete lack of 21-OH function (salt-wasting, SW), and those with partial impairment of 21-OH (simple virilizing, SV) (White et al. 1987).

The affected enzyme, 21-OH, is encoded by an active gene, CYP21, located on the short arm of chromosome 6, with an adjacent inactive pseudogene $(C Y P 21 P)$. Many mutations responsible for the disease have been described (Rodrigues et al. 1987; Higashi et al. 1988b, 1991; Wu and Chung 1991). Most of these mutations are results of gene conversion events between the functional $C Y P 21$ gene and the pseudogene $(C Y P 21 P)$ acting as a reservoir of mutations (Higashi et al. 1988a). Approximately $90 \%$ of the cases are caused by nine specific point mutations and deletions in the $C Y P 21$ gene: $P 30 L, 655 \mathrm{C} / \mathrm{A}>\mathrm{G}$ in intron 2 (i2g), del 8-bp in exon 3, II72N, triple substitution in exon 6 (E6 cluster), V281L, 1761insT, Q318X, and R356W. We designed rapid and convenient PCR-based methods to screen for these known mutations in the CYP21 gene and characterized 34 Japanese patients with 21-OH deficiency.

\section{Materials and methods}

Patients

Thirty-four Japanese CAH patients, including three siblings, were studied. There was no consanguinity between the parents of these patients. In 18 families (20 patients), DNA samples from the parents also were analyzed. Twenty-six patients suffered from the salt-wasting (SW) form of the disease and 8 from the simple virilizing (SV) form. No patient was classified as having the nonclassical form. The patients were diagnosed on the basis of an elevated plasma 17-hydroxyprogesterone. CAH with SW was characterized by the onset of hyperkalemia, hyponatremia, dehydration, and shock requiring treatment with both mineralocorticoids and glucocorticoids. The SV form was identified by the presence of ambiguous genitalia in females. 
After informed consent was obtained from the patients and/or their parents, genomic DNA was prepared from their peripheral blood leukocytes.

PCR amplification and digestion of PCR products

Polymerase chain reaction (PCR) amplification reactions were carried out in 50- $\mu$ l reaction mixtures containing $50 \mathrm{mM} \mathrm{KCl}, 10 \mathrm{mM}$ TRIS- $\mathrm{HCl}, \mathrm{pH} 8.3,1.5 \mathrm{mM} \mathrm{MgCl}_{2}$, $0.01 \%(\mathrm{~W} / \mathrm{V})$ gelatin, $200 \mu \mathrm{M}$ of each dNTP, $1 \mu \mathrm{M}$ of each nucleotide primer, $1 \mu \mathrm{g}$ of genomic DNA, and 2.5 units Taq DNA polymerase (TaKaRa, Kyoto, Japan). The PCR products were digested with appropriate restriction enzymes (New England Biolabs, Beverly, MA, USA) according to the manufacturer's protocol.

\section{Detection of $C Y P 21$ gene deletions}

Deletion of the CYP21 gene was detected by means of PCR with primers 1 and 5 (Table 1) and subsequent digestion with TaqI restriction enzyme (Ogawa et al. 1993). Although the PCR products $(210 \mathrm{bp})$ could be a mixture of both $C Y P 21$ and $C Y P 21 P$ sequences, a $T a q \mathrm{I}$ restriction site is present only in $C Y P 21 P$ (Fig. 1). Therefore, in normal subjects, equal-intensity 210 - and 187-bp bands could be detected. If one of the CYP21 genes was deleted, the intensity of the 210-bp band decreased relative to that of the 187-bp band. If both the CYP21 genes were deleted, only the 187 bp band could be detected.

\section{Amplification of the CYP21 gene}

Genomic DNA was amplified in two segments by PCR with primers 1 and 2 (fragment 1 ) and 3 and 4 (fragment 2), which selectively amplify the CYP21 gene (Table 1). Primers 2 and 3 were specific for sequences in the nonpseudogene DNA (Tajima et al. 1993b). Fragment 1 represented a 952-bp segment extending from exon 1 to exon 3. Fragment 2 was 2070-bp and extended from exon 3 to beyond exon 10 . Thirty cycles of amplification were used, each consisting of denaturation for $30 \mathrm{~s}$ at $94^{\circ} \mathrm{C}$, annealing for $30 \mathrm{~s}$ at $60^{\circ} \mathrm{C}$ (primer $1 / 2$ ) or $64^{\circ} \mathrm{C}$ (primer $3 / 4$ ), and extension for $30 \mathrm{~s}$ (primer $1 / 2$ ) or $120 \mathrm{~s}$ (primer $3 / 4$ ) at $72^{\circ} \mathrm{C}$. If the sample had the $C Y P 21$ deletion or an 8-bp deletion in exon 3 (del 8-bp), no PCR products were generated with these primers.

Detection of seven point mutations

For the second round of PCR, $1 \mu \mathrm{l}$ of the PCR product from each first-stage amplification was used with the appropriate primers listed in Table 1 . Thirty cycles of amplification were used, each consisting of a denaturation step for $30 \mathrm{~s}$ at $94^{\circ} \mathrm{C}$, an annealing step for $30 \mathrm{~s}$ at $58^{\circ}$ or $60^{\circ} \mathrm{C}$, and an extension step for $30 \mathrm{~s}$ at $72^{\circ} \mathrm{C}$. Using fragment 1 as a template, we performed a second round of PCR to detect the P3OL and $i 2 g$ mutations. Fragment 2 was used as a template to detect the $I 172 N$, E6 cluster, V281L, Q318X, and R356W mutations. The volume of the reaction mixture for PCR was $50 \mu \mathrm{l}$ and $5 \mu \mathrm{l}$ of each PCR product was incubated for at least $2 \mathrm{~h}$ with 5-10 units of the appropriate restriction enzyme. Electrophoresis was performed using a 3\%-6\% agarose gel and visualized by ethidium bromide staining.

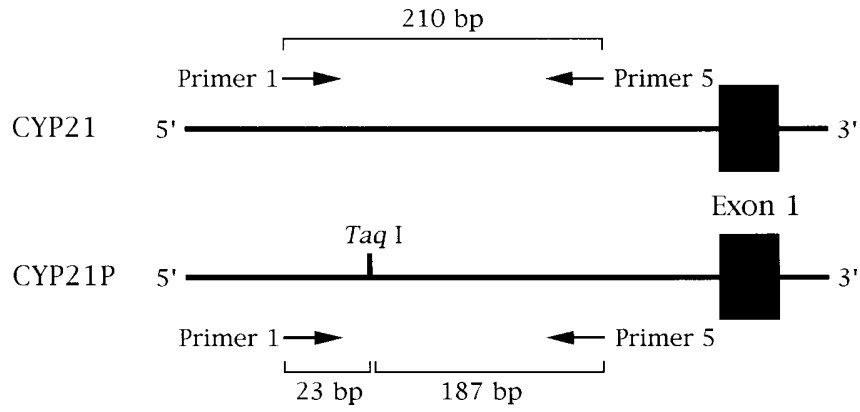

Fig. 1. Locations of primer 1 and 5 in CYP21 and CYP21P. A TaqI restriction site is present only in CYP21P

Table 1. PCR primers used for amplification of $C Y P 21$ gene

\begin{tabular}{|c|c|c|}
\hline Primer 1 & $-231--211$ & 5'-TGC ATT TCC CTT CCT TGC TTC-3' \\
\hline Primer 2 & $+701-+721$ & 5'-GCA GGG AGT AGT CTC CCA AGG-3' \\
\hline Primer 3 & $+701-+721$ & 5'-CCT TGG GAG ACT ACT CCC TGC-3' \\
\hline Primer 4 & $+2749-+2770$ & $5^{\prime}$-AGG GGT TCG TAC GGG AGC AAT A-3' \\
\hline Primer 5 & $-42--22$ & $5^{\prime}-\overline{\mathrm{CTG} \text { AGG TGC CAC TTA TAG CTC- } 3^{\prime}}$ \\
\hline Primer 6 & $+67-+88$ & $5^{\prime}$-AAG CTC CGG AGC CTC CAC CTC $\boldsymbol{G}-3^{\prime}$ \\
\hline Primer 7 & $+195-+214$ & 5'-AGA TCA GCC TCT CAC CTT GC-3' \\
\hline Primer 8 & $+524-+547$ & 5'-TGG GGC ATC CCC AAT CCA GGT CCC-3' \\
\hline Primer 9 & $+656-+677$ & 5'-ACC AGC TTG TCT GCA GGA GGA $\boldsymbol{T}$-3' \\
\hline Primer 10 & $+1000-+1020$ & 5'-TCT CCG AAG GTG AGG TAA CA $T$ - $3^{\prime}$ \\
\hline Primer $11 b$ & $+1375-+1395$ & $5^{\prime}$-AGC TGC ATC TCC ACG ATG TGA-3' \\
\hline Primer 11a & +1375-+1397 & 5'-TCA GCT GCT TCT CCT CGT TGT GG-3' \\
\hline Primer 12 & +1373-+1396 & 5'-GAT CAC ATC GTG GAG ATG CAG CTG-3' \\
\hline Primer 13 & $+2129-+2153$ & $5^{\prime}$-TGG GCC GTG TGG TGC GGT GGG GCA A-3 \\
\hline Primer 14 & +1975-+1993 & 5'-CCA GAT TCA GCA GCG ACT G-3' \\
\hline Primer 15 & $+2113-+2136$ & $5^{\prime}$-TGG GGC AAG GCT AAG GGC ACA AC $\boldsymbol{C}-3^{\prime}$ \\
\hline
\end{tabular}

Underlined primers are antisense primers

Italic letters indicate modified nucleotides 
$V 281 L$ and $Q 318 X$ mutations

Because these mutations abolish restriction enzyme recognition sites, we performed nested PCR using primers 12 and 13, followed by ApaLI or PstI digestion. When the $V 281 L$ mutation is present, the $A p a \mathrm{LI}$ restriction site is lost, and when the $Q 318 X$ mutation is present, the Pst I site is lost. Digestion of the PCR products with ApaLI yielded 375-, 311-, and 95-bp fragments if the $V 281 L$ mutation was absent, and 686- and 95-bp fragments if the mutation was present. Digestion with PstI yielded 299-, 204-, 158-, and 120-bp fragments if the $Q 318 X$ mutation was absent, and 457-, 204-, and 120-bp fragments if the mutation was present.

\section{$P 30 L, i 2 g, I 172 N$, and $R 356 W$ mutations}

We developed a rapid, modified PCR assay using mismatch primers to detect these mutations.

\section{P30L mutation}

Using primers 6 and 7, a 148-bp fragment containing the $89 \mathrm{C}>\mathrm{T}$ mutation site was amplified. The mismatched $\mathrm{G}$ in primer 6 introduced an AccII restriction site in the normal sequence. Digestion of the PCR product with AccII yielded 126- and 22-bp fragments if the mutation was absent and a 148-bp fragment if the mutation was present.

\section{$i 2 g$ mutation}

A modified PCR was performed for detection of the $655 \mathrm{C} /$ A $>$ G mutation using primers 8 and 9. Primer 9 was designed to introduce a Sau3AI restriction site in the mutant PCR products. Digestion of the PCR product yielded a 156bp fragments if the mutation was absent and 133- and 23-bp fragments if the mutation was present.

\section{I172N mutation}

Using primers 3 and 10, we performed nested PCR to detect the $999 \mathrm{~T}>$ A mutation. Primer 10 was designed to introduce an NdeI site into the normal products. Digestion of the PCR products with NdeI yielded a 320-bp fragment if the mutation was absent and 297-and 23-bp fragments if the mutation was present.

\section{R356W mutation}

A modified PCR amplification was performed to detect the $R 356 W$ mutation using primers 14 and 15 . Primer 15 was designed to introduce an Eco52I restriction site in the normal PCR products. Digestion of the PCR product yielded a 162-bp fragment if the mutation was present and a 136-bp fragment if the mutation was absent.
E6 cluster mutation

E6 cluster mutations were analyzed by means of allelespecific PCR. Two PCRs were performed, one reaction using primers 3 and $11 \mathrm{~b}$ detected the normal allele, and the other with primers 3 and 11a detected the mutant allele. If the sample was normal and homozygous, then a product would be generated only from the reaction containing the normal primer. Conversely, if the sample was homozygous mutant, a product would be generated only from the reaction containing the mutant primer. If, however, the sample was heterozygous, products would be generated from both the normal and mutant reactions (Wilson et al. 1995).

\section{1ins $T$ mutation}

When none of the seven mutations or deletions could account for one of the diseased alleles, we sequenced exon 7 of the CYP21 gene from the patient to determine whether there was an insertion of $\mathrm{T}$ at nucleotide 1761 (1761ins T).

\section{Direct sequencing}

Patients 29 and 30 (siblings) were screened for the most common known mutations, but none of these mutations were detected. We further analyzed these patients by direct sequencing of all the exons of the $C Y P 21$ gene. The primers used to amplify each exon of $C Y P 21$ are listed in Table 2. For the amplification of exon 3, the first stage of PCR was performed using primers 8 and $11 \mathrm{~b}$ (specific for the active gene). All nested PCR products were directly sequenced on a Pharmacia LKB A.L.F. DNA Sequencer (Uppsala, Sweden) using a Thermo-Sequenase cycle sequencing kit (Amersham, Buckingham Shire, UK).

\section{Prenatal diagnosis}

Prenatal diagnosis was requested from the family of patient 15 , who was homozygous for the $i 2 g$ mutation. After obtaining informed consent, whole blood was collected from her parents and sister. Chorionic villus sampling was performed at 12 weeks gestation. DNA was extracted by a standard procedure and analyzed by a modified PCR as described in Materials and methods.

\section{Results}

The distribution of the mutations detected in $C Y P 21$ is shown in Table 3. Among the 68 chromosomes, $56(82 \%)$ were characterized by screening for the seven most common point mutations and deletions. Both chromosomes were affected by one of these mutations in 25 (73\%) patients, $6(18 \%)$ patients had only one copy of one of these mutations, and $3(9 \%)$ patients harbored none of the tested mutations. The most frequent mutations were $i 2 g(26 \%)$, 
Table 2. PCR primers used for direct sequencing

\begin{tabular}{lc} 
Exon 1 & \\
$\quad$ Primer rev-1 & $-45--26$ \\
Primer KS-2 & $+244-+261$ \\
Exon 2 & \\
$\quad$ Primer rev-3 & $+257-+274$ \\
Primer KS-4 & $+452-+470$ \\
Exon 3 & $+605-+624$ \\
$\quad$ Primer rev-5 & $+853-+873$ \\
Primer KS-6 & \\
Exon 4-5 & $+890-+907$ \\
Primer rev-7 & $+1239-+1257$ \\
Primer KS-8 & \\
Exon 6 & $+1270-+1288$ \\
$\quad$ Primer rev-9 & $+1471-+1490$ \\
Primer KS-10 & \\
Exon 7 & $+1520-+1540$ \\
$\quad$ Primer rev-11 & $+1808-+1825$ \\
Primer KS-12 & \\
Exon 8 & $+1923-+1940$ \\
Primer rev-13 & $+2211-+2229$ \\
Primer KS-14 & \\
Exon 9 & $+2177-+2196$ \\
Primer rev-15 & $+2374-+2391$ \\
Primer KS-16 & \\
Exon 10 & $+2701-+2721$ \\
Primer rev-17 & $+2749-+2770$ \\
Primer KS-18 & \\
\hline Aniser &
\end{tabular}

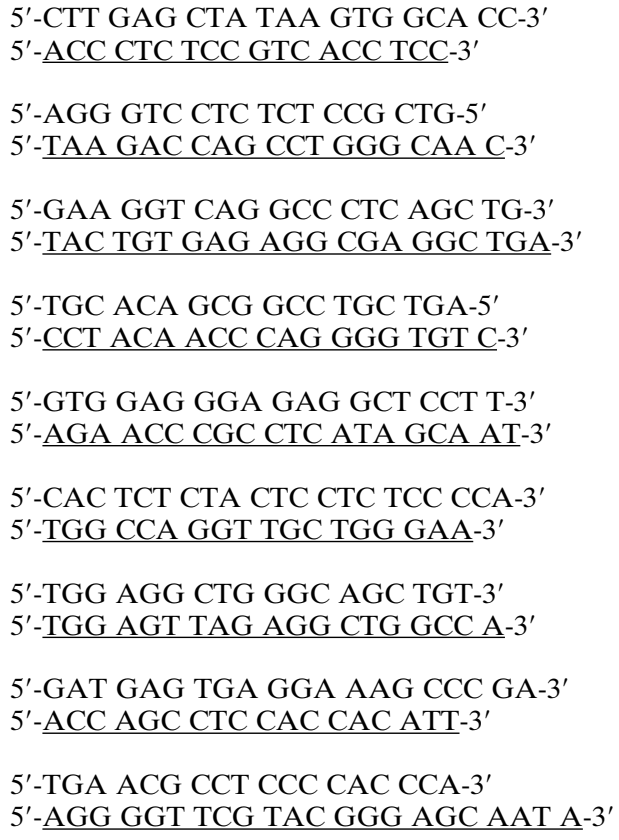

Antisense primers are underlined

Table 3. Distribution of deletions, large gene conversions, and nine common mutations in 34 Japanese CAH patients

\begin{tabular}{lrrr}
\hline & SW & SV & Total \\
\hline P30L & 0 & 1 & 1 \\
i2g & 15 & 3 & 18 \\
del 8 - bp & 0 & 0 & 0 \\
I172N & 2 & 6 & 8 \\
E6 cluster & 1 & 0 & 1 \\
V281L & 0 & 0 & 0 \\
1761 ins $T$ & 0 & 0 & 0 \\
Q318X & 6 & 0 & 6 \\
R356W & 9 & 3 & 12 \\
C.A. & 2 & 0 & 2 \\
Deletion & 7 & 1 & 8 \\
Not detected & 10 & 2 & 12 \\
$\quad$ Total & 52 & 16 & 68 \\
\hline
\end{tabular}

$\mathrm{CAH}$, congenital adrenal hyperplasia

${ }^{a}$ Complex allele: I172N + R356W and V281L + 1761insT + Q318X

$R 356 W(18 \%)$, a deletion of the CYP21 gene (12\%), I172N (12\%), and Q318X (9\%). The P30L, E6 cluster, and 1761ins $T$ mutations were rare, and the del $8-b p$ in exon 3 was not detected in this study. The distribution of the mutation frequencies in Japanese populations does not differ significantly from those previously reported (Speiser et al. 1992), except for the frequency of the deletion of the CYP21 gene. The frequency of a deletion of the CYP21 gene in Japan is lower than in Western countries $(20 \%-$ 30\%) (Wilson et al. 1995; Speiser et al. 1992; Wedell et al. 1994). Two families in this series had one allele with more than one mutation. Patient 19 had the $I 172 N$ and $R 356 W$ mutations on his maternal allele while patient 21 carried the
$V 281 L, 1761$ ins $T$, and $Q 318 X$ mutations on her paternal allele. These complex alleles probably resulted from large gene conversions or multiple mutation events.

Analysis of the segregation of point mutations and deletions in the $18 \mathrm{CAH}$ pedigrees (20 patients) showed three de novo mutations ( $8 \%$ allelic frequency; an $i 2 g$ mutation in patient 17, a $P 30 L$ in patient 31 , and an $R 356 \mathrm{~W}$ in patient 33). The de novo mutation rate for this disease is reportedly low (<1\%) (Wedell et al. 1994; Barbat et al. 1995; Speiser et al. 1994), although it has been reported to be higher in Japanese populations $(\sim 20 \%)$ (Tajima et al. 1993a,b). Our result supports the conclusion that the incidence of de novo mutations among Japanese is high.

Direct sequencing of CYP21 in patients 29 and 30 revealed homozygous $S 268 T$ and N493S mutations (Fig. 2). Their parents were heterozygous for these two mutations. The $S 268 T$ mutation has been reported to be a normal polymorphism (Rodrigues et al. 1987; Wu and Chung 1991), although the functional effect of the N493S mutation has never been analyzed. Barbat et al. (1995) described the N493S mutation as a disease-causing mutation, but Wedell and Luthman (1993) considered this change to be normal polymorphism. Ordonez-Sánchez et al. (1998) found a very high frequency of the $N 493 S$ mutation in a Mexican population, and the proportion of homozygosity for the N493S substitution was higher for patients. Rodrigues et al. (1987) also reported a patient in whom a hemizygous $N 493 S$ mutation was combined with the $S 268 T$ mutation. It is possible that the N493S substitution may result in decreased enzymatic activity only when combined with the effect of the S268T mutation, and that these two changes appear in linkage disequilibrium. A synergistic effect of partially inacti- 
vating mutations has already been documented for the CYP21 gene (Nikoshkov et al. 1997).

The DNA analysis of a fetus from family 15 revealed that only one of the chromosome 6 alleles carried the $i 2 g$ mutation (Fig. 3), predicting that the fetus would be unaffected. After delivery, this prediction was confirmed by postnatal DNA analysis and hormonal studies.

\section{Discussion}

We have designed a coordinated strategy to detect the nine most common 21-hydroxylase mutations. Depending upon the mutation to be detected, we applied one of two simple strategies: digestion of PCR-amplified gene fragments with appropriate restriction enzymes or the use of modified PCR methods employing mismatch primers followed by restriction analysis. In contrast to previous methods, such as dot blot analysis, single-strand conformation polymorphism (using radioactive probes), or allele-specific PCR (which amplifies normal and mutant alleles in different tubes) (Speiser et al. 1992; Ogawa et al. 1993; Tajima et al. 1993b; Wilson et al. 1995), this strategy could characterize six common CYP21 gene mutations (P30L, i2g, I172N, V281L, $Q 318 X$, and $R 356 \mathrm{~W}$ ) by using ethidium bromide-stained agarose gel and six common restriction enzymes. Because we could not develop a modified PCR approach for the E6 cluster mutation, we applied allele-specific PCR to detect this mutation. Using these rapid and convenient PCR-based methods, we successfully screened $34 \mathrm{CAH}$ patients. Our results indicate that most $(76 \% ; 52 / 68)$ of the CAH mutations may be detected by screening for five mutations $(i 2 g$, $I 172 N, Q 318 X, R 356 W$, and deletion of $C Y P 21)$.

The most frequent mutations found in the SW form of the disease were $i 2 g$ (29\% of the SW chromosomes), $R 356 \mathrm{~W}$ (17\%), deletion (13\%), and Q318X (11\%). In the SV form, $I 172 N$ was very frequent (37\% of the SV chromosomes). Phenotype-genotype correlations may be drawn by considering the phenotype of patients homozygous or hemizygous for these mutations (Table 4). Individuals homozygous or hemizygous for $i 2 g$, the E6 cluster, $Q 318 X$, and $R 356 W$ had an SW phenotype, suggesting that these mutations were responsible for the SW phenotype. Conversely, those who were homozygous or hemizygous for the $I 172 N$ mutation had an SV phenotype. However, the phenotypes of the patients who were compound heterozygotes could not be predicted from their genotypes with complete certainty. For instance, patients whose genotypes were $i 2 \mathrm{~g} / \mathrm{R} 356 \mathrm{~W}$ could have either the SV or SW phenotype (see Table 4).

Patient 9, who was clinically diagnosed as having SW, had an affected younger brother (patient 10) who had not developed salt-losing symptoms. Similarly, patient 7, who did not have SW, had a younger sister (not shown in Table 4) who died of hypovolemic shock. Indeed, the mutation $i 2 \mathrm{~g}$, which leads to aberrant splicing, was found in both clinical forms. This may be explained by differences in the rate of correct splicing in the adrenals of the patients (White and New 1992; White et al. 1994). It is also possible that the a)

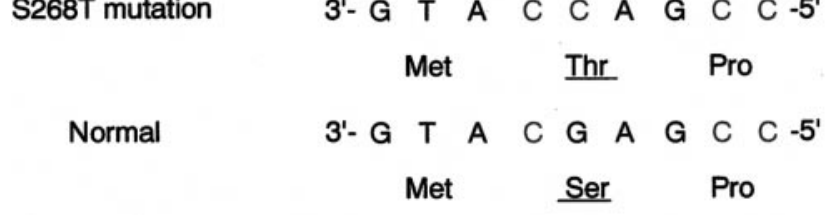

b)

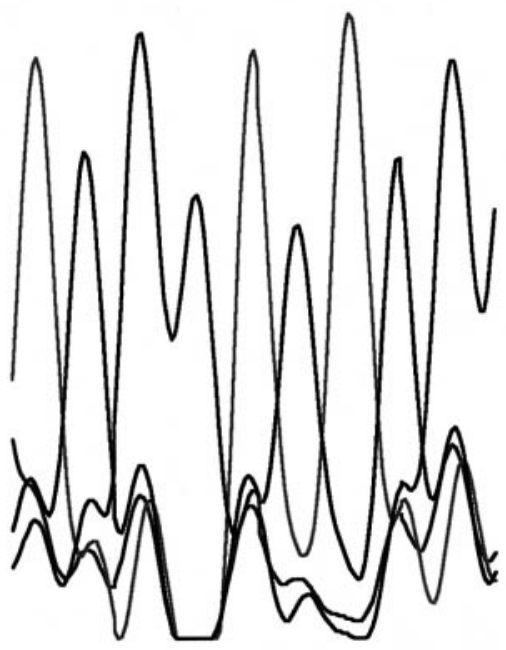

N493S mutation

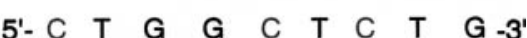

N493 mutation
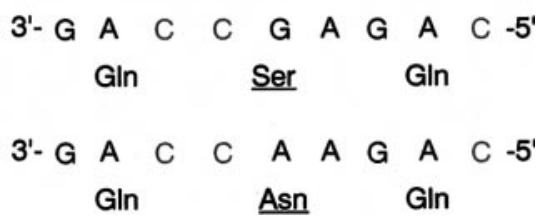

Fig. 2a, b. Direct sequence analysis of genomic DNA from patient 29 (antisense strand). Homozygous S268T (a) and N493S (b) mutations were detected

phenotype may depend on individual variations, a term that must include not only other genetic factors but also nongenetic factors such as environmental stress.

Since the introduction of neonatal screening for $\mathrm{CAH}$, most children are diagnosed before salt-wasting symptoms develop (Pang et al. 1988). We have found that genotyping is a very useful tool for predicting disease severity in $\mathrm{CAH}$ 

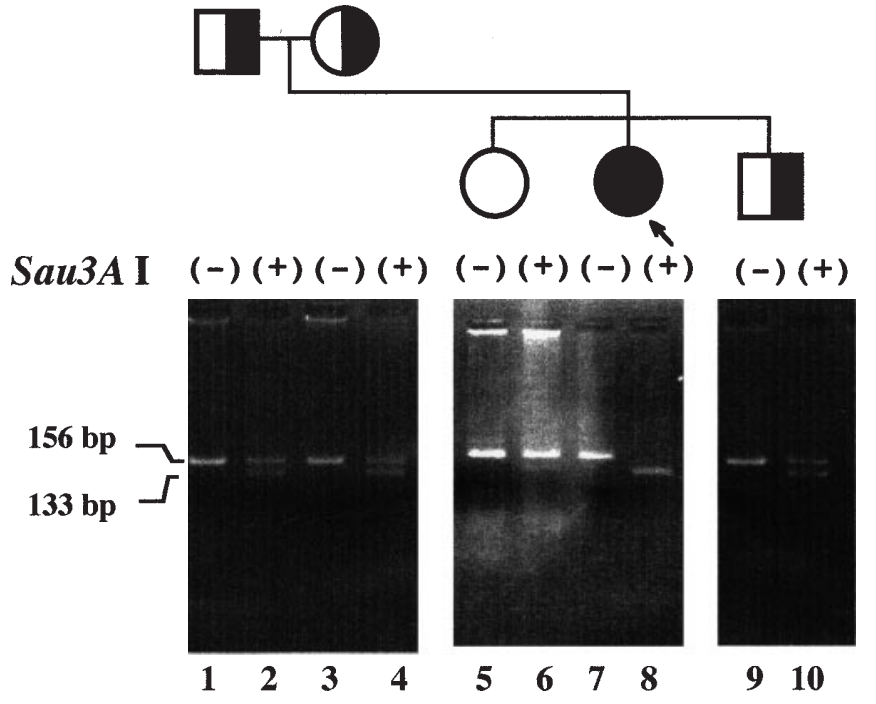

Fig. 3. DNA analysis was performed by $\mathrm{PCR}$ as described in Materials and methods. Patient 15 (lanes 7,8 ) is a homozygote for the $i 2 \mathrm{~g}$ mutation. The patient's father (lanes 1,2) and mother (lanes 3,4) both are heterozygotes for this mutation; her sister (lanes 5,6) is normal. A fetus (lanes 9,10 ) is a heterozygote for this mutation

patients. However, caution is needed when analyzing the $C Y P 21$ genes to avoid misinterpretation of the genotype data because the $C Y P 21$ gene in patients with $\mathrm{CAH}$ may have undergone considerable variations in the gene arrangement as well as in the number of point mutations. With proper vigilance, however, a rapid and accurate diagnosis of CAH can be made by typing the $C Y P 21$ mutations.

In conclusion, PCR-based screening methods are useful in genotyping the Japanese 21-OH-deficient population. Our results showed a close, but not complete, correlation between the molecular defect and the clinical expression of this disease. Therefore, the methods described here are suitable for genetic screening, including the prenatal diagnosis of disease.

\section{References}

Barbat B, Bogyo A, Raux-Demay MC, Kuttenn F, Boué J, SimonBouy B, Serre JL, Boué A, Mornet E (1995) Screening of CYP21 gene mutations in 129 French patients affected by steroid 21-hydroxylase deficiency. Hum Mut 5:126-130

Higashi Y, Tanae A, Inoue H, Fujii-Kuriyama Y (1988a) Evidence for frequent gene conversion in the steroid 21-hydroxylase P-450(C21) gene: implications for steroid 21-hydroxylase deficiency. Am J Hum Genet 42:17-25

Higashi Y, Tanae A, Inoue H, Hiromasa T, Fujii-Kuriyama Y (1988b) Aberrant splicing and missense mutations cause steroid 21-hydroxylase [P-450(C21)] deficiency in humans: possible gene conversion products. Proc Natl Acad Sci USA 85:7486-7490

Higashi Y, Hiromasa T, Tanae A, Miki T, Nakura J, Kondo T, Ohura T, Ogawa E, Nakayama K, Fujii-Kuriyama Y (1991) Effects of individual mutations in the $\mathrm{P}-450(\mathrm{C} 21)$ pseudogene on the $\mathrm{P}-450(\mathrm{C} 21)$ activity and their distribution in the patient genomes of congenital steroid 21-hydroxylase deficiency. J Biochem (Tokyo) 109:638-644

Nikoshkov A, Lajic S, Holst M, Wedell A, Luthman H (1997) Synergic effect of partially inactvating mutations in steroid 21-hydroxylase
Table 4. Genotype-phenotype correlation

\begin{tabular}{lll}
\hline Genotype & Phenotype & Patient no. \\
\hline i2g/i2g & SW & $5,6,8,15$ \\
i2g/del & SW & 28 \\
E6 cluster/del & SW & 27 \\
Q318X/Q318X & SW & 2,12 \\
R356W/R356W & SW & 11 \\
R356W/del & SW & 1 \\
I172N/I172N & SV & 16 \\
I172N/del & SV & 25 \\
i2g/R356W & SW & $9,23,32$ \\
i2g/R356W & SV & 7,10 \\
\hline
\end{tabular}

deficiency. J Clin Endocrinol Metab 82:194-199

Ogawa E, Ohura T, Igarashi Y, Narisawa K, Tada K (1993) Genetic analysis of classical 21-hydroxylase deficiency using polymerase chain reaction and allele-specific oligonucleotide hybridization. Clin Pediatr Endocrinol 2:125-132

Ordonez-Sánchez ML, Ramírez-Jiménez S, López-Gutierrez AU, Riba L, Gamboa-Cardiel S, Cerrillo-Hinojosa M, AltamiranoBustamante N, Calzada-León R, Robles-Valdés C, Mendoza-Morfin F, Tuié-Luna MT (1998) Molecular genetic analysis of patients carrying steroid 21-hydroxylase deficiency in the Mexican population: identification of possible new mutations and high prevalence of apparent germ-line mutations. Hum Genet 102:170-177

Pang S, Wallace MA, Hofman L, Thuline HC, Dorche C, Lyon ICT, Dobbins RH, Kling S, Fujieda K, Suwa S (1988) Worldwide experience in newborn screening for classical congenital adrenal hyperplasia due to 21-hydroxylase deficiency. Pediatrics 81:866-874

Rodrigues NR, Dunham I, Yu CY, Carroll MC, Porter RR, Campbell RD (1987) Molecular characterization of the HLA-linked steroid 21hydroxylase B gene from an individual with congenital adrenal hyperplasia. EMBO J 6:1653-1661

Speiser PW, Duont J, Zhu D, Serrat J, Buegeleisen M, Tusie-Luna MT, Lesser M, New MI, White PC (1992) Disease expression and molecular genotype in congenital adrenal hyperplasia due to 21-hydroxylase deficiency. J Clin Invest 90:584-595

Speiser PW, White PC, Dupont J, Zhu D, Mercado AB, New MI (1994) Prenatal diagnosis of congenital adrenal hyperplasia due to 21-hydroxylase deficiency by allele-specific hybridization and southern blot. Hum Genet 93:424-428

Tajima T, Fujieda K, Fukii-Kuriyama Y (1993a) De novo mutation causes steroid 21-hydroxylase deficiency in one family of HLA-identical affected and unaffected siblings. J Clin Endocrinol Metab 77:86-89

Tajima T, Fujieda K, Nakayama K, Fujii-Kuriyama Y (1993b) Molecular analysis of patient and carrier genes with congenital steroid 21-hydroxylase deficiency by using polymerase chain reaction and single strand conformation polymorphism. J Clin Invest 92:2182-2190

Wedell A, Luthman H (1993) Steroid 21-hydroxylase (P450c21): a new allele and spread of mutations through the pseudogene. Hum Genet 91:236-240

Wedell A, Thilén A, Ritzén EM, Stengler B, Luthman H (1994) Mutational spectrum of the steroid 21-hydroxlase gene in Sweden: implications for genetic diagnosis and association with disease manifestation. J Clin Endocrinol Metab 78:1145-1152

White PC, New MI (1992) Genetic basis of endocrine disease 2: congenital adrenal hyperplasia due to 21-hydroxylase deficiency. J Clin Endocrinol Metab 74:6-11

White PC, New MI, Dupont B (1987) Congenital adrenal hyperplasia (first of two parts). N Engl J Med 316:1519-1524

White PC, Tusie-Luna MT, New MI, Speiser PW (1994) Mutations in steroid 21-hydroxylase (CYP21). Hum Mut 3:373-378

Wilson RC, Wei JQ, Cheng KC, Mercado AB, New MI (1995) Rapid deoxyribonucleic acid analysis by allele-specific polymerase chain reaction for detection of mutations in the steroid 21-hydroxylase gene. J Clin Endocrinol Metab 80:1635-1640

Wu DA, Chung BC (1991) Mutations of P-450c21 (steroid 21-hydroxylase) at $\mathrm{Cys}^{428}, \mathrm{Val}^{281}$, and $\mathrm{Ser}^{268}$ result in complete, partial, or no loss of enzymatic activity, respectively. J Clin Invest 88:519-523 